\title{
Economía E INTEgRación EN UNA América LATINA QUE SIGUE BUSCANDO LA ESTABILIDAD. UNA APROXIMACIÓN
}

\author{
Alfredo Guerra-Borges
}

$\mathrm{E}$ n los años ochenta, abrumada América Latina por la crisis de la deuda externa, la integración pasó al refrigerador. Nadie quería hablar de ella pues se la consideraba cosa del pasado. Lo interesante es que ahora que los tiempos presentes recuerdan vivamente aquellos años tormentosos de la crisis, se habla mucho de integración pues se la considera cosa del futuro. ¿Qué explica este cambio de actitud? ¿Qué pasó en la economía y la integración latinoamericana entre aquellos años y los actuales? En estos apuntes ensayaremos algunas reflexiones sobre el panorama actual de la integración, pero como la integración no tiene autonomía de vuelo y su alcance depende de las condiciones generales de las economías que se asocian, examinaremos también someramente lo que ha acontecido en la economía latinoamericana en los últimos veinte años.

\section{Los años de la expiación}

A principio de los años ochenta las economías latinoamericanas ingresaron todas a un periodo de pro-

Alfredo Guerra-Borges, Instituto de Investigaciones Económicas, Universidad Nacional Autónoma de México. funda crisis económica, después de dos décadas de crecer a tasas anuales acumulativas muy significativas. La deuda externa acumulada en el decenio anterior había llegado a un punto difícil de sobrellevar y los términos de intercambio de los productos de exportación se habían desplomado, en algunos países al nivel de los años de la crisis mundial de los años treinta. Al elevarse verticalmente los precios de los hidrocarburos en la década anterior, los gobiernos se resistieron a ajustar las economías a las condiciones del nuevo entorno y optaron por contratar deuda externa. En un principio todo fue cosa de pedir y recibir; los bancos andaban sueltos buscando por doquier dónde colocar nuevos créditos, incluso a tasas reales negativas, pues la liquidez internacional era abundante. Pero en los ochenta la situación cambió bruscamente, las tasas de interés se elevaron en forma desmedida y los bancos cerraron la llave del crédito. Se inauguró el ciclo de las renegociaciones de la deuda y del pago de su caudaloso servicio.

Pero también había que corregir muchas cosas que andaban mal. Tenía que abatirse la inflación, que en algunos países tenía niveles estratosféricos; tenían que corregirse los abultados déficit fiscales, 
el innecesario abultamiento del Estado; el desequilibrio de las balanzas de pagos y era imperioso recuperar la estabilidad. En suma, había que recrear las condiciones de operación de las economías a fin de ajustarlas a un nuevo escenario internacional que estaba definido, como siempre, en los países centrales; ajustado, como siempre, a los requerimientos de las economías de éstos; presidido, como siempre, por la disponibilidad en ellos de nuevos avances tecnológicos que les confieren una supremacía insuperable.

Por la expiación de los desaciertos y los excesos del pasado se tuvo que pagar un alto precio; al final de los ochenta los países mostraban señales de estar exhaustos y aunque por entonces comenzaron a recuperarse ya no recuperaron el dinamismo de las décadas anteriores. En resumen, puede decirse que el proceso de ajuste se prolongó hasta 1990. En el periodo 1981-1983 se impuso un ajuste excesivo que por su brutalidad redundó en una considerable reducción del producto nacional y una caída aún mayor de la demanda interna. Entre 1984 y 1987 se recuperaron tanto el producto como la demanda interna, pero para volver a caer en 1988 y 1989. El Sol pareció volver a salir en 1991-1994 en que volvieron a crecer la producción y la demanda interna, las exportaciones aumentaron de manera significativa, aunque menos que las importaciones; se recuperó la inversión y hubo una nueva afluencia de capitales externos, sobre todo como acompañamiento de la privatización de las empresas públicas. ${ }^{1}$ Lamentablemente, el desempeño de la economía latinoamericana en 1995-2000 desvaneció toda ilusión en que aquella recuperación fuera el principio de la estabilidad. En ese quinquenio las dos principales economías de América Latina sufrieron crisis devastadoras, la de México en 1995 y la de Brasil en 1998, y Argentina entró de manera inexorable en la pendiente que la arrastró al peor desastre que ha conocido América Latina.

\section{Los diez mandamientos}

Con la crisis de los ochenta entró en crisis también el paradigma desarrollista y en su lugar se instauró el paradigma neoliberal, que el economista John Williamson resumió en diez prescripciones a las que denominó, con inusitada fortuna, Consenso de Wasbington. En apretado resumen esas diez prescripciones eran las siguientes: en materia de disciplina fiscal el déficit debía llevarse al mínimum; en gasto público el objetivo debía ser la reducción y eliminación de los subsidios, reorientando el gasto hacia la educación, la salud y la infraestructura; en materia de reforma tributaria debía ampliarse la base tributaria y garantizar tasas marginales moderadas; en materia crediticia y cambiaria el compromiso debía ser contar con tasas de interés reales y positivas determinadas por el mercado de cada país y tipos de cambio "competitivos"; la liberalización comercial debía orientarse a la reducción al mínimo de las tarifas y no aplicarlas jamás a bienes intermedios requeridos en la producción de exportación; la inversión extranjera directa debía estimularse firmemente; las empresas públicas debían privatizarse y el Estado debía acometer con mano firme la desregulación de la economía. El décimo mandamiento, finalmente, disponía que los derechos de propiedad fueran reforzados. En rigor de verdad, éste no era un punto esencial del paradigma. Como el propio John Williamson aclaró, oportunamente, la inclusión de los derechos de propiedad tuvo por objeto completar el decálogo.

Se ha dicho que "las ideas sobre la manera de hacer próspero un país han sido siempre caprichosas. El decenio transcurrido no ha sido diferente de los precedentes más que en cuanto a la variedad y la volatilidad de las prescripciones económicas que gozaban del favor de los académicos, los asesores políticos y los segmentos mejor informados de la 
población mundial. Pero su especificidad se debe a la supuesta existencia de un acuerdo unánime sobre las políticas que deben aplicar los países pobres para llegar a ser ricos". 2 Ese acuerdo hipotéticamente consentido en forma unánime fue el Consenso de Washington; aunque como la realidad es más fuerte que la teoría, los gobiernos aplicaron de manera diferente y con distinta intensidad y apego sus diferentes mandamientos.

¿Qué convirtió al Consenso de Washington en una verdadera "marca internacional" que causó furor en los años ochenta? En primer lugar, la crisis y posterior hundimiento del sistema soviético a fines de los ochenta desacreditó la planificación y de modo bastante confuso toda forma de intervención del Estado en la economía. En segundo lugar, la crisis de la deuda externa tenía atolondrados a los políticos y gobernantes latinoamericanos; en tercer lugar, el Consenso sintetizaba el pensamiento dominante de Washington, la capital victoriosa. Y en cuarto lugar, lo que hizo irresistible el producto fue la decisión del Banco Mundial y el Fondo Monetario Internacional de subordinar sus préstamos a la adopción de políticas inspiradas en el Consenso.

Había además otra razón, aparte de las ya mencionadas, que sonaba más familiar a los oídos latinoamericanos. Como apunta el Secretario General de la CEPAL, "la liberalización económica fue presentada al mundo en desarrollo como la respuesta a estrategias ineficientes asociadas a la protección comercial, a los altos niveles de intervención estatal y a la captación de rentas por parte de los agentes económicos que dichas estrategias generaban. Fue presentada también como la forma de aprovechar plenamente las oportunidades que ofrecía la globalización ....”3

Ahora ya han transcurrido quince años y causa desasosiego que al finalizar los años noventa una institución liberal como el Diálogo Iberoamericano haya concluido su evaluación del periodo con las siguientes palabras: "Al finalizar la década, América Latina seguía mostrando mayor desigualdad que cualquier otra región del mundo en cuanto a la distribución del ingreso y de los activos (incluida la tierra)". Tan impresionante conclusión es por completo coincidente con el balance a que llegaron el Banco Interamericano de Desarrollo (BID) y la Comisión Económica para América Latina (CEPAL) en sus informes del año 2000. ${ }^{5}$ De acuerdo con el BID, en un conjunto de seis regiones del mundo por su nivel de ingreso per cápita, América Latina ocupó en 2000 el quinto lugar, después de los países desarrollados, el sudeste asiático, los países del Medio Oriente y los países de Europa del Este. Sólo los países del resto de Asia y África tienen ingresos menores. En cambio, hace medio siglo América Latina sólo era superada por los países actualmente desarrollados.

El BID agrega que "aparte de moderado, el crecimiento económico ha sido notablemente inestable..." Si bien la inestabilidad del crecimiento es un problema crónico de la región, el BID estima que sus "manifestaciones recientes quizás han sido más notorias y perniciosas que en el pasado”. Además, el BID constata que "en América Latina se encuentran algunos de los países con las peores distribuciones de ingreso en el mundo (...) la mala distribución del ingreso es un rasgo común a toda la región". ${ }^{6}$

El BID informa también que de acuerdo con las encuestas realizadas durante tres años recientes "sólo 10\% de los latinoamericanos cree que la situación económica actual sea buena, mientras un abrumador 60\% considera que sus padres vivían mejor". 7

En resumen, la insatisfacción con los resultados de las reformas es cada vez mayor, y hay razón ciertamente porque los resultados distan mucho de aque- 
lla certeza, aquel aplomo con que los portadores del nuevo paradigma auguraron la curación final del crónico malestar latinoamericano. Sobre este trasfondo de avances y retrocesos es que ha tenido lugar la integración económica en América Latina.

\section{¿Qué hemos hecho en materia de integración?}

Tras el práctico resquebrajamiento de los convenios de integración, suscritos en los años sesenta, a que obligó la crisis de la deuda externa y la drasticidad de los programas de ajuste económico a que hicimos referencia anteriormente, en los años noventa hubo una auténtica carrera en pos de la suscripción de acuerdos de libre comercio, bilaterales unos y multilaterales otros. De hecho, la diversidad no es tan grande como parece a primera vista. El denominador común es que casi todos se limitan al establecimiento de áreas de libre comercio, con la excepción del MERCOSUR y de los convenios históricos (Mercado Común Centroamericano, Comunidad Andina y CARICOM), que, no obstante la multiplicidad de acuerdos bilaterales, son los que constituyen el núcleo duro de la integración latinoamericana.

Indiscutiblemente es un hecho relevante el elevado número de acuerdos suscritos en los últimos años; en apariencia constituye una rectificación histórica del obstinado entorpecimiento de la apertura recíproca de los mercados latinoamericanos que fue característica en los años sesenta y setenta, pero en verdad no es ésta la explicación. Lo que explica la celeridad de la firma de nuevos acuerdos es que desde los años ochenta los gobiernos latinoamericanos emprendieron la apertura unilateral de las economías al resto del mundo. Una vez consumada la apertura al exterior no fue difícil volver los ojos hacia América Latina. Este hecho tiene un signifi- cado especial pues no siendo la proliferación de acuerdos la expresión de una resuelta voluntad política de revaloración de la integración regional, no hay razón para esperar que anime a los gobiernos la voluntad de ampliar y profundizar los tratados para ir más allá del libre comercio.

Un rasgo común de todos los tipos de convenios, particularmente de los bilaterales, es que tienen listas de excepción que a menudo contienen los productos que por su interés significativo los países desean sustraer del libre comercio. De igual manera, las normas de origen son utilizadas muchas veces para restringir el libre comercio. Hay, por tanto, liberalización comercial pero restringida por un latente proteccionismo de los países, lo que no constituye ninguna novedad.

Uno de los problemas más perjudiciales es el de las medidas unilaterales violatorias de la normatividad convenida, o el incumplimiento de los plazos previamente acordados. Pero en esto no hay sorpresas; fue una conducta habitual en el pasado. La principal violación de los convenios suscritos, que no tiene precedente en la experiencia anterior, se ha dado en el Mercado Común Centroamericano, en el que como consecuencia de los convenios suscritos con México en los años noventa los países centroamericanos otorgan a ese país concesiones que no se otorgan entre sí o han fijado aranceles distintos de los establecidos por el régimen arancelario centroamericano. Un segundo caso se tiene en el Tratado de Libre Comercio del Grupo de los Tres (Colombia, México y Venezuela) en el que se hace abstracción del Arancel Externo Común de la Comunidad Andina. Bolivia, por su parte, negoció por separado de la Comunidad con el MERCOSur.

La conclusión bastante ingrata que se puede sacar de lo apuntado es que de no haber mediado la tolerancia de los miembros del Mercado Común 
Centroamericano y la Comunidad Andina, los acuerdos seguramente se hubieran fracturado. Y una segunda conclusión es la señalada por Mónica Hirst, que compartimos, de acuerdo con la cual "el agotamiento de una postura regionalista tal vez constituya el legado político más importante dejado a América Latina por la crisis de la deuda externa y sus efectos sobre la economía de la región". ${ }^{8}$

El resultado positivo de la multitud de convenios suscritos ha sido el incremento del comercio intrarregional, en parte como recuperación de los niveles alcanzados con anterioridad a la crisis de los ochenta, y en parte como nuevo comercio. Los efectos de la liberalización comercial, mucho mayor que en el pasado, podrían ser mayores pero su capacidad de expansión se ve mediatizada por la inestabilidad económica a que se ha hecho referencia con anterioridad. Conviene tener en cuenta, por otra parte, que los convenios de libre comercio no han dado origen a un comercio propiamente intralatinoamericano pues, al igual que en el pasado, las principales corrientes de intercambio son las que existen entre países vecinos (Guatemala y El Salvador en el Mercado Común Centroamericano, Colombia y Venezuela en la Comunidad Andina y Argentina y Brasil en el MerCosur).

El proceso de mayor importancia y trascendencia en la integración latinoamericana es sin duda el Mercado Común del Sur (MERCosur). En primer lugar, porque nació bajo el impulso de Brasil y Argentina, dos de los tres países más importantes de América Latina. En segundo lugar, la sola presencia de Brasil, que es por sí solo la octava potencia industrial del mundo, imprime al MERCosur una proyección mundial y un peso específico que no ha tenido jamás ningún otro convenio de integración latinoamericana. En tercer lugar, su crecimiento y consolidación han sido sostenidos desde que se dieron los primeros pasos en 1986. Ahora, sin embargo, el proceso pasa por una crisis originada ante todo por la crisis de Argentina, sus severas repercusiones en Uruguay y los problemas que aquejan a la economía brasileña, agudizados por las expectativas y especulaciones en relación con las elecciones presidenciales.

\section{¿Qué es realmente lo nuevo en la integración latinoamericana?}

Lo realmente nuevo es un Estados Unidos interesado en la integración comercial. Este hecho, revelado a fines de los ochenta, rompe radicalmente la posición tradicional de ese país: privilegiar el multilateralismo. No es que ahora lo haya abandonado, sigue teniendo para Estados Unidos una importancia primordial. Lo nuevo es que ahora conduce paralelamente su política multilateralista en la Organización Mundial de Comercio, como en el pasado lo hizo en el GATT, y su política de integración comercial con los países del Hemisferio Occidental. El primer paso se dio en 1985 al iniciar las negociaciones de un Acuerdo de Libre Comercio con Canadá, ocasión en que el ex presidente Ronald Reagan anunció la intención de avanzar hacia la formación de un bloque comercial que, según sus palabras, comprendería “desde el Yukón hasta Yucatán". Los dos pasos siguientes los dio en 1990 el ex presidente Bush, padre del presidente actual, pero de dimensión política, experiencia y personalidad incomparables con las disminuidas de su hijo. Ese año, el 11 de junio, los ex presidentes Bush y Salinas de Gortari anunciaron su decisión de establecer una zona de libre comercio entre Estados Unidos y México. Y el 27 de junio el señor Bush dio a conocer su Iniciativa para las Américas, consistente en la constitución a largo plazo de una zona hemisférica de libre comercio. El cuarto 
paso, esta vez el decisivo y propiamente hemisférico, lo dio el ex presidente Clinton en diciembre de 1994 al proponer y conseguir de todos los países del Hemisferio, excepto Cuba, que fue excluida, la firma de un compromiso de encaminarse a la creación del Área de Libre Comercio de las Américas en el año de gracia de 2005. Esta es la secuencia de la evolución de una nueva actitud de Estados Unidos hacia la integración que cambió por completo los escenarios de la misma.

Los principales episodios de esta nueva historia han sido, por una parte, la lucha política del Ejecutivo con el Congreso de Estados Unidos por conseguir la autorización para negociar, la cual le fue negada durante ocho años, hasta fines de 2002, pocos días antes de las vacaciones de los congresistas, en agosto.

Sin pérdida de tiempo, el representante comercial de Estados Unidos, señor Robert Zoellick, pasará ahora a negociar sendos acuerdos comerciales con Chile y Singapur, y enseguida con Centroamérica y Marruecos. Esta aparente dispersión da lugar a preguntarse: ¿Cuál es la lógica que preside la política de negociaciones comerciales del actual gobierno de Estados Unidos?... Ante todo hay que tener en cuenta que el presidente Bush es, antes que nada, un hombre de negocios, su carrera es la de un ejecutivo de alto nivel y no de un político y, en consecuencia, considera que su misión principal es abrir mercados para las exportaciones de Estados Unidos. En segundo lugar, la administración Bush concibe la negociación de cada convenio más como ejercicios para sentar precedentes que como acuerdos comerciales por derecho propio, enfoque que representa un regreso a la estrategia que siguió James Baker en los años ochenta. En tercer lugar, no puede extrañar por lo anterior que el presidente Bush haya nombrado representante comercial de Esta- dos Unidos al señor Zoellick, quien fue brazo derecho del señor Baker cuando éste se desempeñó como Secretario del Tesoro durante la presidencia de Ronald Reagan y Secretario de Estado durante el mandato de George Bush padre.

Para ilustrar el caso cabe hacer referencia a la negociación del Tratado de Libre Comercio de Estados Unidos y Canadá, de 1986 a 1987. Para entonces el principal objetivo de Estados Unidos era abrir negociaciones en el GATT sobre los llamados "temas nuevos" (servicios, inversiones y derechos de propiedad intelectual), pero esta iniciativa encontró gran resistencia de otros miembros del GATT. La estrategia de Baker fue entonces blandir la amenaza de "volverse bilateral" si sus socios comerciales eran renuentes a aceptar los acuerdos multilaterales en los temas indicados. La estrategia dio resultado, se aceptó la discusión en el GATT de los "temas nuevos” y de paso se suscribió el tratado con Canadá.

El gobierno de Estados Unidos tiene objetivos comerciales que se propone alcanzar en la Ronda de Negociaciones de la Organización Mundial de Comercio, acordadas en 2001, en Qatar. Y en esta dirección adquiere importancia estratégica primordial la negociación y suscripción del ALCA. Las negociaciones con Chile y con Centroamérica son peldaños de la escalera que conduce al ALCA y además son formas de presión sobre los países que, como Brasil, oponen resistencia a negociar el ALCA en las condiciones de Estados Unidos. De igual manera, negociar y obtener concesiones de 34 países hemisféricos en el ALCA es mucho más fácil que conseguirlas de 140 países en la OMC, por lo que el ALCA puede constituir un fuerte impulso a extender estas concesiones en el orden multilateral.

¿Qué significa el ALCA para América Latina y cuáles son las condiciones actuales para su negociación, una vez concedida al gobierno estadounidense la Ley 
de Autoridad de Promoción Comercial (TPA, por sus siglas en inglés)? De acuerdo con lo convenido en Miami por los Jefes de Estado y de Gobierno en 1994, el principio básico de las negociaciones sería alcanzarla "sobre la base de los acuerdos regionales y bilaterales existentes (...) haciéndolos más parecidos". Sin embargo, Estados Unidos pretendió desde las primeras reuniones ministeriales que el ALCA se alcanzara mediante la adhesión individual de cada país al Tratado de Libre Comercio de América del Norte (TLCAN). A esto se opuso el MERCOSUR y en la Tercera Reunión Ministerial de Belo Horizonte consiguió que tal pretensión fuera desechada. La otra victoria del MERCosur en Belo Horizonte fue que en vez de la pretensión de Estados Unidos y otros países de suscribir acuerdos con anticipación al año 2005, señalado como término de las negociaciones, se aprobó el principio de que nada estará negociado hasta que todo esté negociado, lo que en la jerga de las negociaciones comerciales se conoce como "principio del single undertaking". Posteriormente, el MERCOSUR consiguió que en la última y crucial etapa de las negociaciones, Brasil y Estados Unidos tendrán la copresidencia. Esto consagra lo que desde la segunda reunión ministerial celebrada en Cartagena había manifestado el BID, que en un documento de la época afirmaba que tan incomprensible era un ALCA sin Brasil y el MERCOSUR como un ALCA sin Estados Unidos y el TLCAN.

Así las cosas, ahora que el término formal de las negociaciones está muy cercano, el escenario parece haberse oscurecido, una vez más, por los avatares de la economía. Estados Unidos está en recesión. El presidente Bush y su Secretario del Tesoro habían venido declarando que había llegado a su fin el periodo recesivo, sobre todo con la vista puesta en las elecciones parlamentarias de noviembre de 2002, pero una y otra vez se han tenido que revisar las estimaciones. La revisión que hizo el FMI, en el Informe sobre las Perspectivas Mundiales, que presentara en septiembre de 2001, bajó la tasa de crecimiento de Estados Unidos a 2.1\%, y la de Europa 1.1\%. Japón seguiría sumido en la crisis que lo agobiaba desde hacía unos cinco años. En todo el mundo, sólo Corea tendría un crecimiento de 4\%, lo que de nuevo llama la atención sobre el modelo de desarrollo que ha seguido ese país muy distante, por no decir lo opuesto, al Consenso de Washington.

Por supuesto, para 2005 o un poco más, cuando las negociaciones del ALCA lleguen a su término, la economía de Estados Unidos se habrá recuperado, ¿pero puede decirse lo mismo de América Latina?... Actualmente, desde los círculos oficiales en el norte como en los gobiernos del sur, se habla con justificado temor y aprehensión de que América Latina parece haber vuelto a la etapa de las crisis. La de Argentina es casi inconcebible. Fue el más fiel de todos los alumnos del FM; nadie como Argentina llegó tan lejos en la observación de su breviario. Sin embargo, se estimaba que su crecimiento, en el 2002, sería, aproximadamente, de $-6 \%$, y que $51 \%$ de su población estaría bajo la línea de pobreza, una constatación desgarradora pues Argentina fue desde el siglo pasado el país latinoamericano de más alto nivel de producto por habitante.

La crisis de Brasil fue aún más amenazadora. Aunque el FMI le otorgó un crédito de 30 mil millones de dólares, nadie apostaba a que eso bastase. Y si eso no bastaba, la Secretaría del Tesoro de Estados Unidos y el propio FMI se preguntaron (sin cuidarse de que no trascendiera al público) qué otra arma podrían emplear cuando al parecer se estaban quedando sin cartuchos.

La peor noticia para la negociación del ALCA es, sin duda, el recrudecimiento del proteccionismo en Estados Unidos. En primer lugar, por la elevación 
de las tarifas de importación del acero. Aun más perjudicial, y esta vez la amenaza es para toda América Latina, es el nivel nunca antes alcanzado del proteccionismo en el caso de la agricultura. La nueva ley de subsidios a la agricultura le otorgará en los seis años venideros subsidios por un total de 190 mil millones de dólares, lo que abaratará aún más las exportaciones agrícolas de Estados Unidos. Brasil y Argentina decidieron ya llevar el asunto a la consideración de la OMC.

Hay que agregar que en materia de protección a la agricultura los países desarrollados, ante todo Estados Unidos, la Unión Europea y Japón, se cierran cada vez más. En total los países desarrollados subsidian la agricultura a razón de 1 mil millones de dólares diarios, o sea, un total de 350 mil millones de dólares en el año, cifra que es siete veces mayor que el total de recursos de los programas de ayuda a los países en desarrollo.

Con Brasil en crisis y el MERCOSUR en crisis, ¿cómo negociar? Y sin ellos el ALCA es un ejercicio sin mayor trascendencia para Estados Unidos. Para este país sigue siendo lo más importante el área de América del Norte. La primera visita concedida por el presidente Bush a un jefe de Estado fue la del Primer Ministro canadiense. Y la primera visita que hizo Bush al extranjero fue a México. El comercio de México con Estados Unidos supera el comercio de todos los demás países de América Latina con Estados Unidos. Y Canadá es el principal socio comercial de Estados Unidos en el mundo. Esto lo dice todo.

¿Qué pasará? ¿Cuál será la posición negociadora del presidente de Brasil? ¿Cómo saldrá Argentina de su profunda crisis? ¿Además de Argentina, de Brasil y de Ecuador, qué otros países vendrán a confirmar que se ha vuelto a la etapa de las crisis?... No hay lugar para razonar las conjeturas, debo terminar. Pero me estremece tener que hacerlo con las palabras que Ignacio Ramonet escribió al hacer su propia apreciación del panorama mundial contemporáneo: "en el umbral del III milenio cada uno puede constatar que la incertidumbre ha llegado a ser la única certidumbre".

\section{Notas}

${ }^{1}$ Ver CEPAL, 15 años de desempeño económico, América Latina y el Caribe, 1980-1995, Santiago de Chile, 1996.

${ }^{2}$ Moisés Naim, “Avatars du Consensus de Washington”, Le Monde Diplomatique, mars 2000, París. Del mismo autor, “Washington Consensus or Washington Confusion?", Foreign Affairs, spring 2000, Carnegie Enfowment for International Peace, Washington D.C.

${ }^{3}$ José Antonio Ocampo, Retomar la agenda del desarrollo, Santiago de Chile, 2001.

${ }^{4}$ Nancy Birsdall y Augusto de la Torre, El Disenso de Washington, Interamerican Dialogue, Washington, DC, 2000, p.15 (vía Internet).

${ }^{5}$ BID, Desarrollo: más allá de la economía. Informe 2000, Washington DC, 2000; y CEPAL, Estudio económico de América Latina y el Caribe, 1999-2000, Santiago de Chile, 2000.

${ }^{6}$ BID, op.cit., pg. VI.

${ }^{7}$ Ibid.

8 Mónica Hirst, "Condicionamiento y motivaciones del proceso de integración y fragmentación en América Latina", en Integración Latinoamericana, Núm. 175, Buenos Aires, Instituto para la Integración de América Latina, 1992.

${ }^{9}$ Ignacio Ramonet, Geopolitique du chaos, París, Gallimard, 2001, p.17. 